\title{
Review Article \\ Cytokine Serum Levels as Potential Biological Markers for the Psychopathology in Schizophrenia
}

\author{
Dimitre H. Dimitrov, Shuko Lee, Jesse Yantis, Craig Honaker, and Nicole Braida
}

South Texas Veterans Health Care Systems, San Antonio, TX 78229-4404, USA

Correspondence should be addressed to Dimitre H. Dimitrov; dimitre.dimitrov@va.gov

Received 25 August 2014; Accepted 27 November 2014; Published 11 December 2014

Academic Editor: Livia Carvalho

Copyright (c) 2014 Dimitre H. Dimitrov et al. This is an open access article distributed under the Creative Commons Attribution License, which permits unrestricted use, distribution, and reproduction in any medium, provided the original work is properly cited.

We discuss the role of immune system disturbance in schizophrenia and especially changes of serum levels of cytokines in patients with schizophrenia. The cytokines are essential to wide range of functions related to the defense of the organisms from infectious and environmental dangers. However it is not known whether cytokines influence the presentation of psychotic symptoms. Identification of changes in the serum level of certain cytokines and their correlation with distinct psychopathological symptoms may facilitate the identification of subgroups of patients who are likely to benefit from immunotherapy or anti-inflammatory therapy. Such patients may benefit from tailored immunotherapy designed for modulation of abnormal cytokine levels related to specific positive or negative symptoms of schizophrenia.

\section{Introduction}

Accumulating evidence supports the view that immunological dysfunction may have a role in the etiology of psychotic disorders. In a recent publication in Nature by the Schizophrenia Working Group of the Psychiatric Genomic Consortium were identified 108 schizophrenia associated loci [1]. Notable associations with the dopaminergic and glutaminergic neurotransmitters as well as associations with voltage gated calcium channels subunits were found. The most significant association was with the major histocompatibility complex and with a region involved in acquired immunity. A recent study provides encouraging evidence that biological signatures for schizophrenia can be identified in blood serum [2]. The role of the immune system disturbance was recently reviewed [3]. An important role leading to these changes is played by the cytokines $[4,5]$.

Cytokines are low-molecular weight proteins secreted by immune cells and other cell types in response to a number of environmental stimuli, particularly infections. They have wide-ranging roles in the innate and adaptive immune systems, where they help regulate the recruitment and activation of lymphocytes as well as immune cell differentiation and homeostasis. In addition, some cytokines possess direct effector mechanisms, including induction of cell apoptosis and inhibition of protein synthesis. Previously we described dysregulated production of cytokines and their association with psychopathology of schizophrenia as well as the possible involvement of the Th17/IL17 pathway $[6,7]$. We found significantly increased levels of GRO, MCP-1, MDC, and SCD40L and significantly decreased levels of IFN- $\gamma$, IL-2, IL12-p70, and IL-17. In addition, we observed positive correlations between levels of cytokines and the Positive and Negative Symptom scale (PANSS) scores in subjects with schizophrenia for G-CSF, IL-1 $\beta$, IL-1ra, IL-3, IL-6, IL-9, IL-10, sCD40L, and $\operatorname{TNF} \beta$. The main objective of this review is to provide further evidence of cytokines linked to severity and duration of schizophrenia as well as with distinct symptoms of psychopathology based on the obtained PANSS scores. Such approach may lead to the discovery of reliable biomarkers for schizophrenia and new immunological therapy designed to control different distinct symptoms of schizophrenia. 


\section{Evidence Obtained on the Basis of the Classical Th1/Th2 Model}

The importance of macrophages and $\mathrm{T}$ lymphocytes and the cytokines produced by them has been highlighted in the macrophage- $\mathrm{T}$ cell theory of bipolar disorder and schizophrenia [8]. T cells are divided into Th8-cytotoxic cells and CD4 cells-T helper cells (Th). According to a model proposed by Mosmann and Coffman [9] the Th cells were divided into Th1 and Th2. Currently two new subtypes of T cells, Th17 and T regulatory cells (Tregs), are emerging as important factors in the etiology of schizophrenia [10].

According to the classical model Thl cells support cellmediated immune responses and activate macrophages via IFN- $\gamma$. Th2 cells support humoral and allergic responses and play a major role in the transformation of B cells into plasma cells which secrete IL-4, IL-5, IL-9, and IL-13 [11]. By analyzing the type 1 and type 2 responses a decreased production of IFN- $\gamma$ and IL- 2 in schizophrenia was reported in vitro reflecting a blunted production of type 1 cytokines $[12,13]$. An activation of the type 2 immune response is also described in schizophrenia including increased levels of IL-4 and IL-10.

The IFN- $\gamma$ is essential for Th1 cellular response. High levels of IFN- $\gamma$ may lead to CNS inflammation and damage to oligodendrocytes, and this may be one of the reasons why in patients with schizophrenia there is a switch from cellular to humoral Th2 immunity. IFN- $\gamma$ is found in neuronal synapses and it may act at the level of the synapse to influence brain function [14]. Alterations in the levels of cytokines and combinations of cytokines can act synergistically or antagonistically. This depends upon the state of the target cells and the combination of doses and temporal sequence of cytokine secretion. Chronic exposure to proinflammatory cytokines may cause premature maturation and stabilization of these synapses. Thus, alterations in the levels of cytokines can profoundly change synaptic efficiency and changes of synaptic efficiency may lead to changes of cytokine level. For example, it was described that IL-6 and IL-2 inhibit the longterm plasticity and the short-term potentiation of neuronal circuits [14]. IL-6 is viewed as a key danger signal initiating the inflammatory cascade. This cytokine together with IFN- $\gamma$ and IL-12 control the Th17 response $[15,16]$. Overproduction of IL-17 may aggravate inflammatory reactions and contribute to tissue damage. We observed lower levels of IL-17 in veterans with schizophrenia who were treated with different antipsychotics. This may be a compensatory mechanism to lessen the extent of injury due to inflammation [7].

In patients with schizophrenia there is also activation of the type 2 immune response with increased production of Ig E and IL-10 [17]. In CSF, IL-10 levels were found to be related to the severity of psychosis [18]. IL-4, the key cytokine of type 2 immune response, is increased in the CSF of juvenile schizophrenic patients [19]. In a recent metaanalysis, 40 studies of the acute phase of schizophrenia were reviewed. High levels of IL- $1 \beta$, IL- 6 , and TGF- $\beta$ were observed, which were considered to be state markers for schizophrenia. Elevated levels of IFN- $\gamma$, TNF- $\alpha$, and soluble IL-2 receptor (sIL-2R) were considered to be trait markers of schizophrenia [20]. Results reported in another meta-analysis suggested that in vivo there are increased peripheral levels of IL-1ra, sIL-2R, and IL-6 [4].

Additionally, the type 1/type 2 imbalance is associated with an activation of astrocytes and an imbalance in the activation of astrocytes/microglial cells [21]. Microglial cells, deriving from peripheral macrophages, secrete preferably type 1 cytokines such as IL-12, while astrocytes inhibit the production of IL-12 and secrete IL-10 [21]. The view of an overactivation of astrocytes in schizophrenia is supported by the findings of increased levels of the calcium binding protein S100B which is considered a marker of astrocyte overactivation [22].

\section{Cytokines Involved in \\ Regulation of Calcium Channels and Formation of Synapses}

Increased serum levels of eotaxin/CCL-11 were described in patients with schizophrenia [23]. The protein sequence of eotaxin is $66 \%$ similar to human MCP-1 and acts on the chemokine receptor CCR3 which is expressed on eosinophils and mast cells. This data is consistent with the idea that preferential activation of Th2 lymphocytes plays a role in the pathogenesis of schizophrenia. Eotaxin is involved in the regulation of the calcium binding proteins such as calcineurin. Calcineurin is linked to receptors for several brain chemicals including NMDA, dopamine, and GABA. Conditioned calcineurin knockout mice exhibit multiple abnormal behaviors related to schizophrenia [24]. Calcineurin in reactive astrocytes plays a key role in the interaction between proinflammatory and anti-inflammatory signals. In quiescent astrocytes inflammatory mediators such as $\mathrm{TNF} \alpha$ recruit calcineurin to stimulate canonical inflammatory pathway involving NF$\kappa \mathrm{B}$. However in reactive astrocytes calcineurin involves antiinflammatory mediators that inhibit NF- $\kappa$ B. These results suggest that calcineurin forms a molecular pathway whereby reactive astrocytes determine the outcome of the neuron inflammatory process by directing it towards either its resolution or its progression [25]. The recent genome-wide study describing associations of schizophrenia with voltage gated calcium channels may outline the possibility that levels of eotaxin and calcium binding proteins could be used as potential markers for schizophrenia [1].

During the neuroinflammatory process of schizophrenia, cytokines like eotaxin appear to facilitate calcium waves. According to this concept if calcium levels are tweaked different neurotransmitters would be expressed. In depression there would be too few calcium waves and in mania and psychosis calcium waves would be fluid and intense. Cannabis may cause ripples of calcium waves and dreamlike states.

Increased eotaxin/CCL11 levels in blood plasma are associated with ageing in mice and humans [26]. Exposing young mice to CCL11 or the blood plasma of older mice decreases their neurogenesis and cognitive performance [26].

Fractalkine is a transmembrane chemokine and is expressed only by neurons, while the fractalkine receptor 
CX3CR1 is exclusively present on microglial cells. Fractalkine (CX3CL1) and its receptor are also involved in immune cell trafficking to the CNS. Mice that lack the receptor for fractalkine have impaired cognitive function and synaptic plasticity. Microglial cells which have receptor for fractalkine are also required to support hippocampal neurogenesis [27]. Fractalkine is recently described as neuronal "off signal" that keeps microglia in resting states. The chemokine CX3CL1 induced chemokine release of CXCL16-CCL2. This interaction involves neurons, microglia, and astrocytes and that represents an endogenous self-protecting mechanism. It was reported that in this way cell damage due to brain ischemia may be limited by counteracting neuronal death due to glutamate excitotoxicity $[28,29]$. The release of CCL2/MCP1 by astrocytes involves synergistic activity of adenosine and adenosine type 3 receptor on astrocytes.

Fractalkine on one hand appears to prevent excess microglial activation in the absence of injury while promoting activation of microglia and astrocytes during inflammatory episodes [30]. Expression levels of fractalkine and its receptor have been found to change in and around the demyelinating lesions that accompany experimental autoimmune encephalomyelitis (EAE) disease progression. In Alzheimer's disease there is increased activation of microglia around amyloid beta plaques. There are data that fractalkine is increased in patients with mild cognitive impairment as compared to healthy controls and the levels of fractalkine were decreased in severe forms of Alzheimer's disease [31].

The fractalkine receptor is synthesized exclusively by microglia and is essential for their survival and migration. Neurons in the brain increase fractalkine production when they are forming synapses. A very interesting observation was that microglia actively engulf synaptic material and play a major role in synaptic pruning during postnatal development in mice [32]. The authors used mutant mice lacking the gene encoding the fractalkine receptor. The mice lacking the fractalkine receptor had significantly greater numbers of synapses leading to increases in the frequency of spontaneous electrical impulses. According to the authors it appears that the developing brain treats unwanted synapses as if they are invading microorganisms and dispatches the microglial cells to survey the state of the synapses and dispose those deemed unwanted and superfluous [32].

\section{Cytokines in the CNS}

Increased inflammatory markers were found in the dorsolateral prefrontal cortex by using SOLiD next generation sequencing to quantify neuroimmune inflammatory transcripts in postmortem brain samples from patients with schizophrenia [33]. By using a two-step factor analysis in this cluster a high mRNA expression of IL- $1 \beta$, IL- 6 , and IL- 8 was reported in 18 individuals and it was concluded that IL- $1 \beta$ is linked to MHC-II-expressing cells in the white matter and that the disease duration had a positive correlation with IL-6 and IL-1 $\beta$ [33]. Elevated IL- $1 \beta$ expression is known to cause increased secretion of IL- 6 from microglia, astrocytes, and neurons and IL-6 may lead to problems of cell migration, which may be one of the reasons for greater density of inhibitory neurons in the white matter of patients with schizophrenia [34]. In a review of the fetal brain cytokine imbalance of schizophrenia it was report that IL- $1 \beta$ is most capable in inducing the conversion of rat mesencephalic progenitor cells into a dopaminergic phenotype and that IL6 is highly efficacious in decreasing the survival of fetal brain serotonin neurons [35]. It is interesting that enhanced levels of IL-10 during prenatal development are sufficient to prevent the emergence of multiple behavior abnormalities [35].

Neuroinflammation is characterized by the activation of the microglial cells which exhibit an increase in the expression of the peripheral benzodiazepine receptor. An 11C radiolabeled isoquinoline is a peripheral receptor ligand and PET imaging was used for the detection of the activated microglial cells [36].

In this report it was demonstrated that there is an increase in the expression of the peripheral benzodiazepine receptor indicative of neuroinflammation and that focal neuroinflammation is a feature of psychosis and not necessarily present in stable schizophrenic patients. The calcium binding protein S100B expressed in activated astrocytes is increased in the serum and plasma of schizophrenia patients and there is a higher binding potential of the radioactive ligand in the hippocampus of schizophrenic patients [36]. Th17 response could be significantly amplified by dopamine. For example, the stimulation of dopamine receptor D5 expressed on dendritic cells can potentiate Th17 immunity [37]. Dopamine receptors expressed on immune cells modulate Th17-mediated inflammation [37] and the Th17-mediated immune response can be attenuated by D1-like receptor antagonists [38].

The inflammatory cytokines may influence tryptophan degradation, leading to elevated levels of the kynurenine metabolites, and as endogenous kynurenic acid modulates the extracellular levels of glutamate and acetylcholine such increases may be of pathophysiologic significance [12].

\section{Autoimmune Diseases and Psychosis}

A range of psychiatric disorders including psychosis have been observed to occur more frequently in some autoimmune diseases such as systemic lupus erythematosus and multiple sclerosis. There is a similarity in the immune pathogenic principles involved in autoimmunity, chronic inflammation, and psychosis [39]. A 30-year population-based register study has shown that having a prior autoimmune disease and a history of hospitalization with infection increased the risk of schizophrenia by $29 \%$ and $60 \%$, respectively [40].

The Th17 lineage is now implicated in a number of autoinflammatory disorders such as rheumatoid arthritis, multiple sclerosis, and psoriasis [41]. It is also implicated in the autoimmune encephalitis and its role in the neuroinflammatory process in multiple sclerosis [42]. Interestingly an IL-17 producing CD8+ T cells (termed Tc17) was discovered in mice and humans. These Tc17 cells can initiate Th17 autoimmunity by supporting Th17 pathogenicity [43]. 
In a subgroup of psychotic patients the high comorbidity with autoimmune and chronic inflammatory conditions suggests a common underlying immune abnormality underlying both conditions. Immune biomarkers might be found in raised monocyte and microglia inflammatory activation patterns together with reduced numbers and reduced proliferation activity of $\mathrm{T}$ cells. In such case high number of $\mathrm{T}$ reg cells may predominate leading to high serum level of sIL2R and inflammatory skewing of T cells in direction of Th1/Th17 with high levels of IL-12 and IFN- $\gamma$ [44].

\section{Review of the IL-17/Th17}

Previously we reported positive correlation between the levels of cytokines and PANSS scores in patients with schizophrenia. Pathway analyses showed these cytokines to be part of the IL-17 pathway [7]. IL-17 has a remarkable homology with herpes virus saimiri and this led to the hypothesis that during evolution the virus captures a portion of the human gene in order to gain survival advantage during infection $[45,46]$. The Th17 responses are very important in host defense but also in promoting chronic inflammation and autoimmunity [16]. Th17 cells appear to have evolved as cells bridging the innate and the adaptive immunity and are specialized for enhanced cell protection from microorganisms that are not well guarded by the Th1 and Th2 immunity. Th17 cells an IL-17 contribute to host defense against bacterial and fungal infections $[15,16]$. Human Th17 cells remain in the body as a long-lived proliferating effector memory $\mathrm{T}$ cells with unique genetic and functional characteristic [47]. It was reported that inflammation in the brain parenchyma occurs only when Th17 cells outnumber Th1 cells [48]. IL-17 increases the level of GRO and MCP-1 [49, 50]. On the other hand the IL17 mediated monocyte migration occurs partially through MCP-1 induction [51].

IL-17 has been shown to induce expression of several cytokines known to contain nuclear factor kappa $(\mathrm{NF}-\kappa \mathrm{B})$ binding sites in their promoters. NF- $\kappa \mathrm{B}$ is the principal transcription factor in the initiation of the inflammatory response. The precise mechanism by which cell generates IL17 is not fully elucidated but it probably involves calcineurin and cyclic AMP. In response to IL-17 neutrophil specific chemokines such as IL-8 and GRO are generated as well as granulopoietic cytokines such as G-CSF and GM-CSF. By acting on macrophages IL-17 stimulates the release of TNF$\alpha[46]$.

In patients with schizophrenia increased levels of sCD40L are associated with endothelial damage and this event triggers the release of inflammatory mediators [52]. In studies of EAE in mice IL-17 disrupts the blood brain barrier tight junctions [53]. In order for the Th17 cells to enter the CNS CCR6 expression on Th17 cells was required in the first wave of Th17 cells that enter the CNS through the epithelial cells of the choroid plexus. After that IL-17 induces inflammatory gene expression in the astrocytes which triggers a second wave of Th-17 cells. Th17 cells then enter the inflamed brain in a CCR6 independent manner leading to explosive inflammatory cascade with the onset of EAE [54]. The low levels of IL-17 in the veterans observed by us could be due to low levels of IFN- $\gamma$ and IL-12 which do not exert enough inhibitory effect on Th17 cells [7]. The inflammatory changes in astrocytes lead to increased levels of IL-10 and decreased levels of IL-12. The decreased levels of IL-12 lead to decreased IL-17 [21, 22]. Recent study described a subpopulation of Th17 cells that are highly pathogenic and can induce EAE in mice [55]. Generation of the pathogenic cells requires IL-23 stimulation following IL- 6 and TGF $\beta$ stimulation [15].

Not all Th17 cells are involved in autoimmune processes. In contrast to the autoimmunity-promoting Th17 cells, thymus derived natural regulatory cells (nTreg) represent a unique population of cells that inhibits $\mathrm{T}$ cell proliferation and autoimmune processes [56]. $\mathrm{T}$ regulatory cells are a component of the immune system that suppresses immune responses of other T cells. This is an important "self-check" built into the immune system to prevent excessive reactions. These cells are involved in shutting down immune responses after they have successfully eliminated invading organisms and also in preventing autoimmunity. Low levels of IL-2 may impair the proliferation of Treg cells and ultimately result in autoimmunity. The low serum levels of IL-2 could lead to proliferation of Th2 response in the presence of IL-4. This would lead to an allergic response with increased levels of IL4 and IL-13 which leads to isotype switching to IgE [11]. The eosinophilia may lead to lower levels of IL-17.

The expansion and proliferation of Treg cells is dependent on the activity of IL-2. IL-2 activates Treg cells to proliferate and differentiate via the IL-2 receptor. Activated Treg cells shed the IL-2 receptors and the shed receptor (sIL-2R) binds IL-2, inactivates it, and so fine-tunes the immune response. Th17 cells and regulatory $\mathrm{T}$ (Treg) cells play opposite roles in autoimmune disease. The Treg cells use Foxp3 transcription factor. The process of differentiation of Th17 requires STAT3 transcription factor. At the same time both cell subsets require TGF- $\beta$ for their development but there is reciprocal regulation in the generation of these cells. While TGF- $\beta$ induces Foxp3 expression, in the absence of IL-6 and IL-21, TGF- $\beta$ will instead induce Th17 differentiation [56].

Tregs were found to have neuroprotective effect, attenuating microglia-mediated inflammation [57]. Microglia can adopt a neuroprotective phenotype upon activation by cytokines such as IL-4. On the other hand activated microglia produce high level of MCP-1 which triggers microglia proliferation and also serves as a microglia-induced neurodegeneration [57].

\section{Emerging Theories for the Etiology of Psychopathology}

By using convergent functional genomics it was proposed that fibronectin is decreased in high hallucination states and high delusional states and also in fibroblasts from schizophrenic patients [58]. In the above report the authors concluded that a decreased fibronectin and increased neuregulin are involved in high delusional states and decreased fibronectin and increased calcyclin S100A6 in high hallucination states. Of interest is the fact that fibronectin is also a top gene for alcoholism. The authors also propose that 
genes involved in cancer, plasticity, and connectivity (cell morphology, cell to cell signaling, and interaction) are prominent players in psychotic disorders. This hypothesis provides encouraging evidence that there may be different biological markers involved in delusions and hallucinations [58].

If the developing brain treats imperfect synapses as if they were invading microorganisms and dispatches the microglial cells to survey the state of the synapses and dispose of those that are unwanted and superfluous, this may suggest a speculative conclusion that an evolutionary process in nature may have selected primitive cellular mechanisms. These mechanisms are involved in the response to damage, insults, and stressors for analogous higher organism level functions (i.e., increased neuregulin and decreased fibronectin and increased S100A6 and decreased APOE). In this view, psychosis becomes the higher organism brain equivalent of cellular dedifferentiation and disconnection, such as occurring in early stages of inflammation, tissue remodeling, and cancer metastasis.

The evolutionary process has also changed the ratio of astrocytes per neuron. In mice the ratio is 0.3 astrocytes per neuron and in humans this ratio is 1.4 astrocytes per neuron [59]. The dramatic increase of astrocytes in the human brain could be a reason for the occurrence of imbalance between astrocytes and microglia. The overactivation of the astrocytes in schizophrenia is supported by the findings of increased levels of S100B [22].

\section{Prospective Immunological Therapy of Schizophrenia}

According to the cytokine model of schizophrenia it is considered that elevated levels of IL- 6 and other proinflammatory cytokines play a key role and cause a wide adverse effect on the brain including facilitation of the dopaminergic sensitization, diminished hippocampal volumes, and impaired glutamatergic functions [5]. According to this model aberrant fetal programming results in elevation of IL-6 level around puberty and when this is reinforced by peripubertal stress they interact with one another leading to emergence of positive and negative symptoms and cognitive deficits. This model leads to the conclusions that immunological immunotherapy leading to opposing the effect of IL-6 may represent a useful strategy for treatment and that the IL-17 pathway is emerging as a major target in autoimmune disease. For example, Tocilizumab is an antiIL-6 receptor antibody that is approved by the FDA for treatment of rheumatoid arthritis in individuals who have not responded to anti-TNF alpha therapy [5]. On the other hand a recent study has demonstrated that inhibition of STAT3 blocks Th17 development and inhibits experimental uveitis [60]. Inhibition of the STSAT3 pathway offers an additional approach to immunotherapy of schizophrenia.

\section{Conflict of Interests}

The authors declare that there is no conflict of interests regarding the publication of this paper.

\section{Acknowledgment}

The authors would like to thank Dr. Kelly Arneman, Ph.D., for editorial assistance.

\section{References}

[1] Schizophrenia Working Group of the Psychiatric Genome Consortium, "Biological insights from 108 schizophrenia-associated genetic loci," Nature, vol. 511, pp. 421-427, 2014.

[2] E. Schwarz, P. C. Guest, H. Rahmoune et al., "Identification of a biological signature for schizophrenia in serum," Molecular Psychiatry, vol. 17, no. 5, pp. 494-502, 2012.

[3] S. Horváth and K. Mirnics, "Immune system disturbances in schizophrenia," Biological Psychiatry, vol. 75, no. 4, pp. 316-323, 2014.

[4] S. Potvin, E. Stip, A. A. Sepehry, A. Gendron, R. Bah, and E. Kouassi, "Inflammatory cytokine alterations in schizophrenia: a systematic quantitative review," Biological Psychiatry, vol. 63, no. 8, pp. 801-808, 2008.

[5] R. R. Girgis, S. S. Kumar, and A. S. Brown, "The cytokine model of schizophrenia: emerging therapeutic strategies," Biological Psychiatry, vol. 75, no. 4, pp. 292-299, 2014.

[6] D. H. Dimitrov, "Correlation or coincidence between monocytosis and worsening of psychotic symptoms in veterans with schizophrenia?" Schizophrenia Research, vol. 126, no. 1-3, pp. 306-307, 2011.

[7] D. H. Dimitrov, S. Lee, J. Yantis et al., "Differential correlations between inflammatory cytokines and psychopathology in veterans with schizophrenia: potential role for IL-17 pathway," Schizophrenia Research, vol. 151, no. 1-3, pp. 29-35, 2013.

[8] R. C. Drexhage, E. M. Knijff, R. C. Padmos et al., "The mononuclear phagocyte system and its cytokine inflammatory networks in schizophrenia and bipolar disorder," Expert Review of Neurotherapeutics, vol. 10, no. 1, pp. 59-76, 2010.

[9] T. R. Mosmann and R. L. Coffman, "TH1 and TH2 cells: different patterns of lymphokine secretion lead to different functional properties," Annual Review of Immunology, vol. 7, pp. 145-173, 1989.

[10] M. Debnath and M. Berk, "Th17 pathway-mediated immunopathogenesis of schizophrenia: mechanisms and implications," Schizophrenia Bulletin, vol. 40, no. 6, pp. 1412-1421, 2014.

[11] D. D. Chaplin, "Overview of the immune response," Journal of Allergy and Clinical Immunology, vol. 125, no. 2, pp. S3-S23, 2010.

[12] N. Muller and M. J. Schwarz, "Immune system in schizophrenia," Current Immunology Reviews, vol. 6, pp. 213-220, 2010.

[13] V. Arolt, M. Rothermundt, K.-P. Wandinger, and H. Kirchner, "Decreased in vitro production of interferon-gamma and interleukin-2 in whole blood of patients with schizophrenia during treatment," Molecular Psychiatry, vol. 5, no. 2, pp. 150$158,2000$.

[14] P. A. Garay and A. K. McAllister, "Novel roles for immune molecules in neural development: implications for neurodevelopmental disorders," Frontiers in Synaptic Neuroscience, vol. 2, pp. 136-162, 2010.

[15] C. T. Weaver, R. D. Hatton, P. R. Mangan, and L. E. Harrington, "IL-17 family cytokines and the expanding diversity of effector T cell lineages," Annual Review of Immunology, vol. 25, pp. 821852, 2007. 
[16] T. Korn, E. Bettelli, M. Oukka, and V. K. Kuchroo, "IL-17 and Th17 cells," Annual Review of Immunology, vol. 27, pp. 485-517, 2009.

[17] M. J. Schwarz, S. Chiang, N. Müller, and M. Ackenheil, "Thelper-1 and T-helper-2 responses in psychiatric disorders," Brain, Behavior, and Immunity, vol. 15, no. 4, pp. 340-370, 2001.

[18] D. P. van Kammen, C. G. McAllister-Sistilli, and M. E. Kelly, "Relationship between immune and behavioral measures in schizophrenia," in Current Update in Psychoimmunology, G. Wiesselmann, Ed., New York, NY, USA, pp. 51-55, Springer, 1997.

[19] B. B. Mittleman, F. X. Castellanos, L. K. Jacobsen, J. L. Rapoport, S. E. Swedo, and G. M. Shearer, "Cerebrospinal fluid cytokines in pediatric neuropsychiatric disease," The Journal of Immunology, vol. 159, no. 6, pp. 2994-2999, 1997.

[20] B. J. Miller, P. Buckley, W. Seabolt, A. Mellor, and B. Kirkpatrick, "Meta-analysis of cytokine alterations in schizophrenia: clinical status and antipsychotic effects," Biological Psychiatry, vol. 70, no. 7, pp. 663-671, 2011.

[21] F. Aloisi, G. Penna, J. Cerase, B. M. Iglesias, and L. Adorini, "IL-12 production by the central nervous system microglia is inhibited by astrocytes," Journal of Immunology, vol. 159, no. 4, pp. 1604-1612, 1997.

[22] M. Rothermundt, P. Falkai, G. Ponath et al., "Glial cell dysfunction in schizophrenia indicated by increased S100B in the CSF," Molecular Psychiatry, vol. 9, no. 10, pp. 897-899, 2004.

[23] A. L. Teixeira, H. J. Reis, R. Nicolato et al., "Increased serum levels of CCL11/eotaxin in schizophrenia," Progress in Neuropsychopharmacology and Biological Psychiatry, vol. 32, no. 3, pp. 710-714, 2008.

[24] T. Miyakawa, L. M. Leiter, D. J. Gerber et al., "Conditional calcineurin knockout mice exhibit multiple abnormal behaviors related to schizophrenia," Proceedings of the National Academy of Sciences of the United States of America, vol. 100, no. 15, pp. 8987-8992, 2003.

[25] A. M. Fernandez, S. Fernandez, P. Carrero, M. Garcia-Garcia, and I. Torres-Aleman, "Calcineurin in reactive astrocytes plays a key role in the interplay between proinflammatory and antiinflammatory signals," The Journal of Neuroscience, vol. 27, no. 33, pp. 8745-8756, 2007.

[26] S. A. Villeda, J. Luo, K. I. Mosher et al., "The ageing systemic milieu negatively regulates neurogenesis and cognitive function," Nature, vol. 477, pp. 90-94, 2011.

[27] A. D. Bachstetter, J. M. Morganti, J. Jernberg et al., "Fractalkine and $\mathrm{CX}_{3} \mathrm{CR} 1$ regulate hippocampal neurogenesis in adult and aged rats," Neurobiology of Aging, vol. 32, no. 11, pp. 2030-2044, 2011.

[28] K. Biber, H. Neumann, K. Inoue, and H. W. G. M. Boddeke, "Neuronal "on" and "off" signals control microglia," Trends in Neurosciences, vol. 30, no. 11, pp. 596-602, 2007.

[29] M. Rosito, C. Lauro, G. Chece et al., "Trasmembrane chemokines CX3CL1 and CXCL16 drive interplay between neurons, microglia and astrocytes to counteract pMCAO and excitotoxic neuronal death," Frontiers in Cellular Neuroscience, vol. 8, article 193, 2014.

[30] G. K. Sheridan and K. J. Murphy, "Neuron-glia crosstalk in health and disease: fractalkine and CX3CR1 take centre stage," Open Biology, vol. 3, no. 1, Article ID 130181, 2013.

[31] T.-S. Kim, H.-K. Lim, J. Y. Lee et al., "Changes in the levels of plasma soluble fractalkine in patients with mild cognitive impairment and Alzheimer's disease," Neuroscience Letters, vol. 436, no. 2, pp. 196-200, 2008.
[32] R. C. Paolicelli, G. Bolasco, F. Pagani et al., "Synaptic pruning by microglia is necessary for normal brain development," Science, vol. 333, no. 6048, pp. 1456-1458, 2011.

[33] S. G. Fillman, N. Cloonan, V. S. Catts et al., "Increased inflammatory markers identified in the dorsolateral prefrontal cortex of individuals with schizophrenia," Molecular Psychiatry, vol. 18, no. 2, pp. 206-214, 2013.

[34] Y. Yang, S. J. Fung, A. Rothwell, S. Tianmei, and C. S. Weickert, "Increased interstitial white matter neuron density in the dorsolateral prefrontal cortex of people with schizophrenia," Biological Psychiatry, vol. 69, no. 1, pp. 63-70, 2011.

[35] U. Meyer, J. Feldon, and B. K. Yee, "A review of the fetal brain cytokine imbalance hypothesis of schizophrenia," Schizophrenia Bulletin, vol. 35, no. 5, pp. 959-972, 2009.

[36] J. Doorduin, E. F. J. de Vries, A. T. M. Willemsen, J. C. de Groot, R. A. Dierckx, and H. C. Klein, "Neuroinflammation in schizophrenia-related psychosis: a PET study," Journal of Nuclear Medicine, vol. 50, no. 11, pp. 1801-1807, 2009.

[37] C. Prado, F. Contreras, H. González et al., "Stimulation of dopamine receptor D5 expressed on dendritic cells potentiates Th17-mediated immunity," Journal of Immunology, vol. 188, no. 7, pp. 3062-3070, 2012.

[38] K. Nakagome, M. Imamura, H. Okada et al., "Dopamine D1-like receptor antagonist attenuates Th17-mediated immune response and ovalbumin antigen-induced neutrophilic airway inflammation," The Journal of Immunology, vol. 186, no. 10, pp. 5975-5982, 2011.

[39] M. E. Benros, P. R. Nielsen, M. Nordentoft, W. W. Eaton, S. O. Dalton, and P. B. Mortensen, "Autoimmune diseases and severe infections as risk factors for schizophrenia: a 30year population-based register study," The American Journal of Psychiatry, vol. 168, no. 12, pp. 1303-1310, 2011.

[40] M. E. Benros, W. W. Eaton, and P. B. Mortensen, "The epidemiologic evidence linking autoimmune diseases and psychosis," Biological Psychiatry, vol. 75, no. 4, pp. 300-306, 2014.

[41] T. A. Moseley, D. R. Haudenschild, L. Rose, and A. H. Reddi, "Interleukin-17 family and IL-17 receptors," Cytokine and Growth Factor Reviews, vol. 14, no. 2, pp. 155-174, 2003.

[42] F. Jadidi-Niaragh and A. Mirshafiey, "Th17 Cell, the new player of neuroinflammatory process in multiple sclerosis," Scandinavian Journal of Immunology, vol. 74, no. 1, pp. 1-13, 2011.

[43] M. Huber, S. Heink, A. Pagenstecher et al., "IL-17A secretion by CD8+ T cells supports Th17-mediated autoimmune encephalomyelitis," Journal of Clinical Investigation, vol. 123, no. 1, pp. 247-260, 2013.

[44] V. Bergink, S. M. Gibney, and H. A. Drexhage, "Autoimmunity, inflammation, and psychosis: a search for peripheral markers," Biological Psychiatry, vol. 75, no. 4, pp. 324-331, 2014.

[45] Z. Yao, W. C. Fanslow, M. F. Seldin et al., "Herpesvirus Saimiri encodes a new cytokine, IL-17, which binds to a novel cytokine receptor," Immunity, vol. 3, no. 6, pp. 811-821, 1995.

[46] J. Witowski, K. Ksiazek, and A. Jörres, "Interleukin-17: a mediator of inflammatory responses," Cellular and Molecular Life Sciences, vol. 61, no. 5, pp. 567-579, 2004.

[47] I. Kryczek, E. Zhao, Y. Liu et al., "Human TH17 cells are longlived effector memory cells," Science Translational Medicine, vol. 3, no. 104, pp. 104-ra100, 2011.

[48] I. M. Stromnes, L. M. Cerretti, D. Liggitt, R. A. Harris, and J. M. Goverman, "Differential regulation of central nervous system autoimmunity by TH1 and TH17 cells," Nature Medicine, vol. 14, no. 3, pp. 337-342, 2008. 
[49] Y. Hu, F. Shen, N. K. Crellin, and W. Ouyang, "The IL-17 pathway as a major therapeutic target in autoimmune diseases," Annals of the New York Academy of Sciences, vol. 1217, no. 1, pp. 60-76, 2011.

[50] F. J. Dumont, "IL-17 cytokine/receptor families: emerging targets for the modulation of inflammatory responses," Expert Opinion on Therapeutic Patents, vol. 13, no. 3, pp. 287-303, 2003.

[51] S. Shahrara, S. R. Pickens, A. M. Mandelin II et al., "IL17-mediated monocyte migration occurs partially through CC chemokine ligand 2/monocyte chemoattractant protein-1 induction," The Journal of Immunology, vol. 184, no. 8, pp. 44794487, 2010.

[52] P. I. Johansson, A. M. Sørensen, A. Perner et al., "High sCD40L levels early after trauma are associated with enhanced shock, sympathoadrenal activation, tissue and endothelial damage, coagulopathy and mortality," Journal of Thrombosis and Haemostasis, vol. 10, no. 2, pp. 207-216, 2012.

[53] H. Kebir, K. Kreymborg, I. Ifergan et al., "Human TH17 lymphocytes promote blood-brain barrier disruption and central nervous system inflammation," Nature Medicine, vol. 13, no. 10, pp. 1173-1175, 2007.

[54] A. Reboldi, C. Coisne, D. Baumjohann et al., "C-C chemokine receptor 6-regulated entry of $\mathrm{TH}-17$ cells into the $\mathrm{CNS}$ through the choroid plexus is required for the initiation of EAE," Nature Immunology, vol. 10, no. 5, pp. 514-523, 2009.

[55] Y. Lee, A. Awasthi, N. Yosef et al., "Induction and molecular signature of pathogenic $\mathrm{T}_{H} 17$ cells," Nature Immunology, vol. 13, no. 10, pp. 991-999, 2012.

[56] T. Yamazaki, X. O. Yang, Y. Chung et al., "CCR6 regulates the migration of inflammatory and regulatory T cells," Journal of Immunology, vol. 181, no. 12, pp. 8391-8401, 2008.

[57] L. R. Frick, K. Williams, and C. Pittenger, "Microglial dysregulation in psychiatric disease," Clinical and Developmental Immunology, vol. 2013, Article ID 608654, 10 pages, 2013.

[58] S. M. Kurian, H. Le-Niculescu, S. D. Patel et al., "Identification of blood biomarkers for psychosis using convergent functional genomics," Molecular Psychiatry, vol. 16, no. 1, pp. 37-58, 2011.

[59] M. Nedergaard, B. Ransom, and S. A. Goldman, "New roles for astrocytes: redefining the functional architecture of the brain," Trends in Neurosciences, vol. 26, no. 10, pp. 523-530, 2003.

[60] C.-R. Yu, Y. S. Lee, R. M. Mahdi, N. Surendran, and C. E. Egwuagu, "Therapeutic targeting of STAT3 (signal transducers and activators of transcription 3 ) pathway inhibits experimental autoimmune uveitis," PLoS ONE, vol. 7, no. 1, Article ID e29742, 2012. 


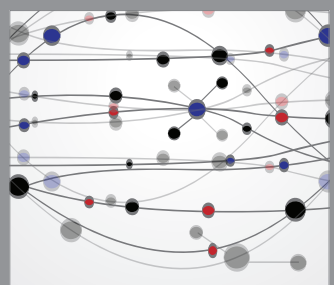

The Scientific World Journal
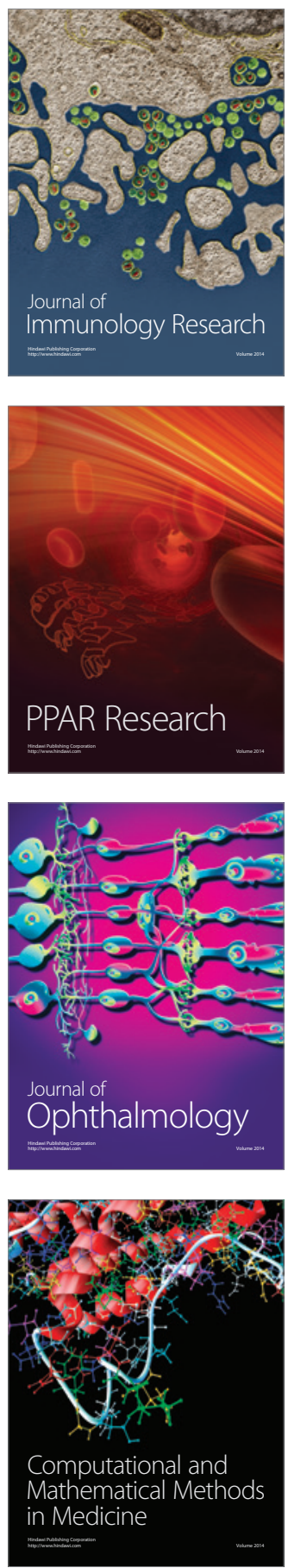

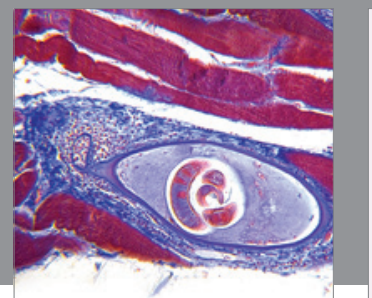

Gastroenterology

Research and Practice
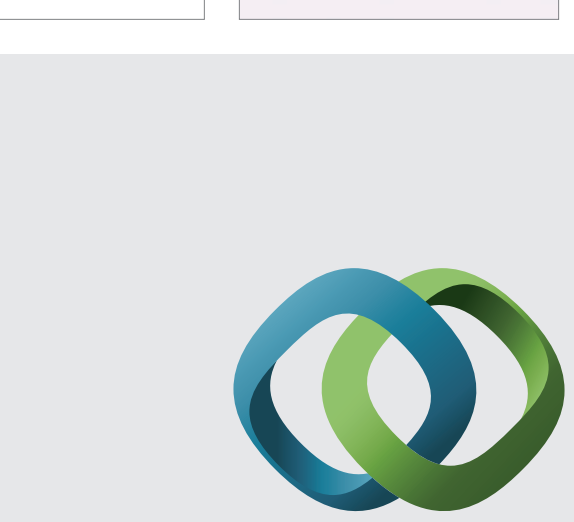

\section{Hindawi}

Submit your manuscripts at

http://www.hindawi.com
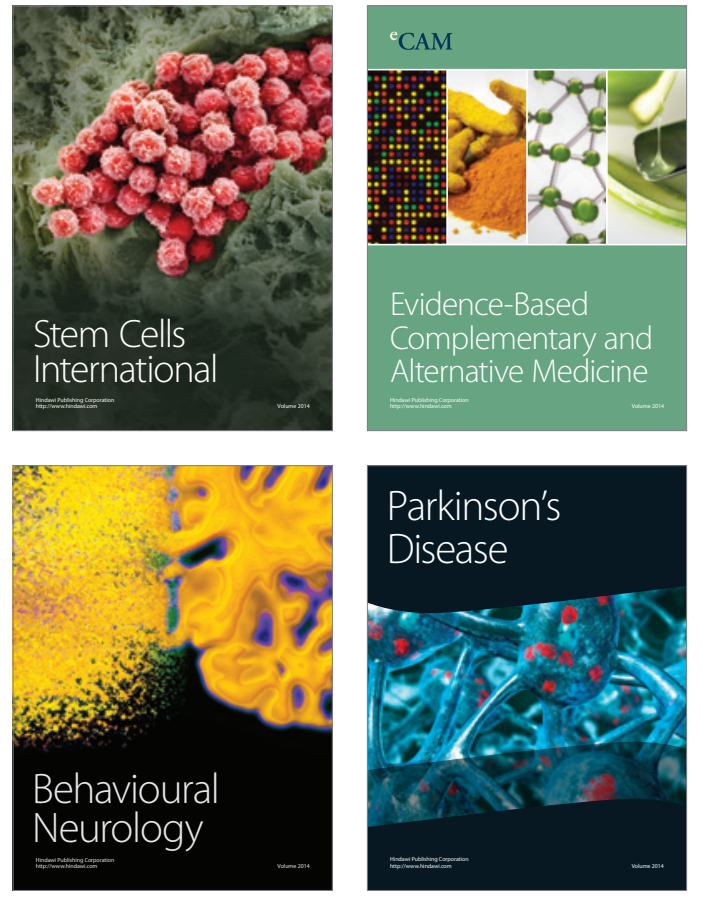
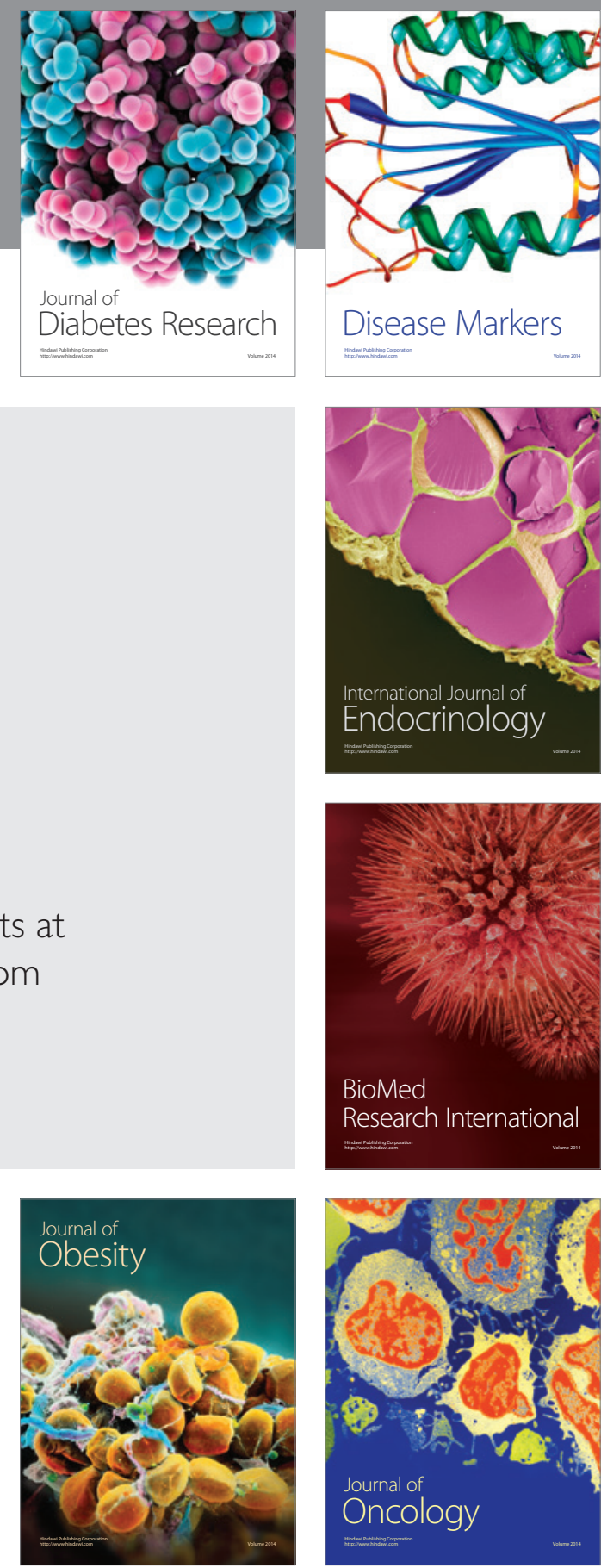

Disease Markers
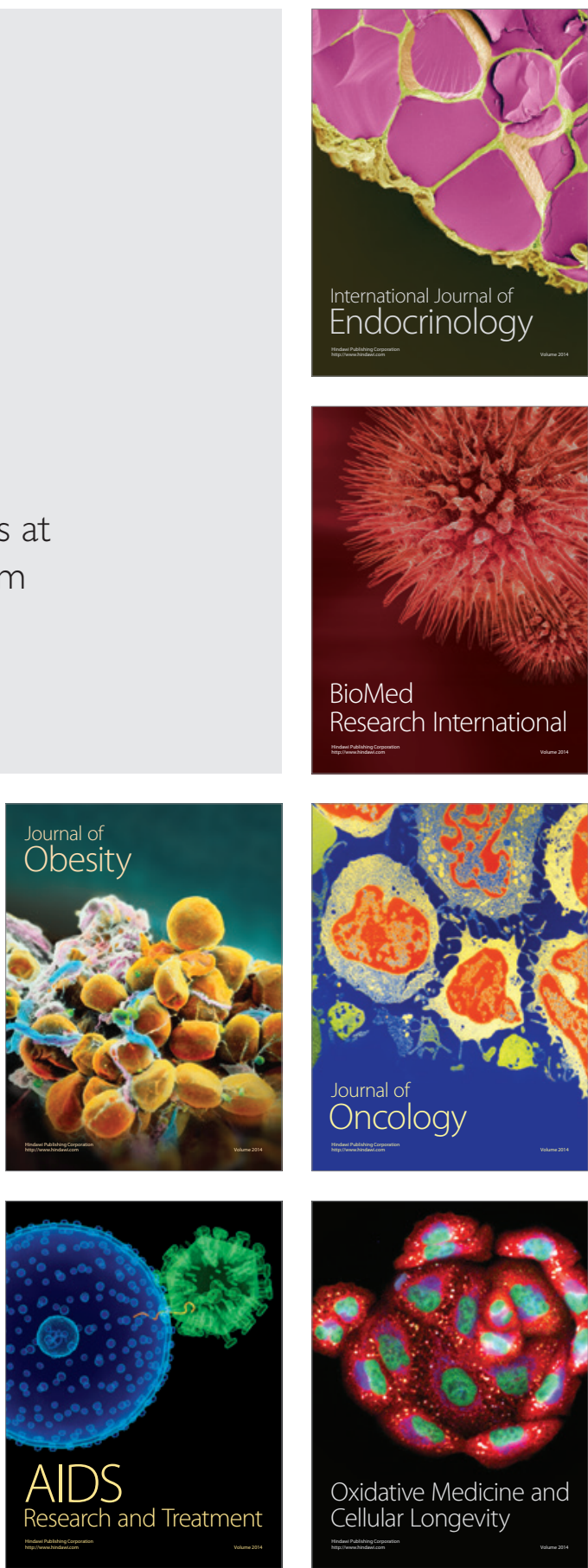